\title{
Evaluation of melatonin efficacy in prevention of bronchopulmonary dysplasia in preterm newborn infants
}

\author{
Manizheh Mostafa Gharehbaghi ${ }^{1 \oplus}$, Sadollah Yeganedoust ${ }^{1 \oplus}$, Elnaz Shaseb $^{2 \odot}$, \\ Majid Fekri ${ }^{1 \odot}$
}

Departments of ${ }^{1}$ Neonatology, and ${ }^{2}$ Clinical Pharmacology, Tabriz University of Medical Sciences, Tabriz, Iran.

\begin{abstract}
Background. Excessive production of reactive oxygen species play an important role in the pathogenesis of bronchopulmonary dysplasia (BPD). Melatonin is an effective antioxidant and free radical scavenger. The aim of this study was to evaluate the efficacy of melatonin in the prevention of BPD in preterm infants with respiratory distress syndrome.

Methods. In a randomized clinical trial, 80 preterm newborn infants with respiratory distress syndrome and gestation age of 27-32 weeks were allocated randomly in two groups. Group A consisted of 40 neonates who received surfactant with the INSURE technique. Patients in group B received melatonin $5 \mathrm{mg} / \mathrm{kg} / \mathrm{day}$ per gastric tube for 3 days in addition to the surfactant. The primary outcome was on the occurrence of BPD. The secondary outcome was considered other complications of prematurity, duration of hospital stay and mortality.
\end{abstract}

Results. The mean gestational age and birth weight of studied patients were $31.3 \pm 3.8$ weeks and $1189 \pm 84$ grams, respectively. Thirty-five (43.8\%) patients were girls and $45(56.2 \%)$ were boys. BPD was diagnosed in 24 (60\%) neonates of group A and 18 (45\%) patients in group B, p=0.02. The duration of hospital stay, need for mechanical ventilation and mortality rate were significantly lower in patients in group B ( $\mathrm{p}=0.02,0.003,0.009$ respectively).

Conclusions. Our study results showed that BPD, mortality and hospital stay reduced with melatonin treatment in preterm infants. However, future studies with a larger number of patients are needed to confirm these beneficial effects.

Key words: melatonin, respiratory distress syndrome, bronchopulmonary dysplasia, preterm infants.

Melatonin is an endogenous indolamine synthesized in the pineal gland from serotonin. ${ }^{1}$ It is an effective antioxidant and free radical scavenger. ${ }^{2}$ Melatonin has a key role in many important physiological functions, such as regulation of circadian rhythms, stimulatory action in the immune system, visual, reproductive, cardiovascular, neuroendocrine and neuroimmunological actions. ${ }^{3}$

Melatonin scavenges free radicals that are oxygen and nitrogen derived metabolites and

$凶$ Manizheh Mostafa Gharehbaghi

gharehbaghimm@yahoo.com

Received 29th April 2021, revised 13th June 2021, 1st August 2021, accept 18th August 2021. protects DNA from the damage induced by them. ${ }^{4}$ Clinically, melatonin has been used in sleep disorders, neurodegenerative diseases, aging and cancers in adults. ${ }^{5}$ In recent years there are few reports about its use in children and neonates. ${ }^{6-8}$

Preterm infants are susceptible to oxidative stress-induced injury because of oxygen use at initial resuscitation and immature fetal antioxidant system. Although oxygen therapy is essential in the management of neonatal respiratory disorders, oxygen exposure, unavoidably leads to excessive production of reactive oxygen species (ROS) in the respiratory system. Its association with peroxidant drugs, concomitent infections or extrapulmonary 
inflammation can promote the depletion of antioxidants and contribute to the development of bronchopulmonary dysplasia (BPD). ${ }^{9}$ Gitto et al. examined melatonin treatment in preterm newborns diagnosed with respiratory distress syndrome (RDS) to determine whether melatonin may delay the development of BPD. ${ }^{10}$ They found newborns with BPD and without melatonin treatment had higher levels of proinflammatory cytokines than those who received melatonin.

The etiology of BPD is multifactorial and factors including mechanical ventilation induced barotrauma, volutrauma, oxygen toxicity, inadequate nutrition, infection and inflammation may contribute to impaired alveolar and vascular development in vulnerable premature lungs. ${ }^{11,12}$ Excess generation of reactive oxygen and nitrogen species multiate molecules and are important mediators of cell and tissue damage. ${ }^{13}$ Various pharmacologic and non pharmacologic approaches have been investigated for prevention and treatment of preterm lung injury and BPD. ${ }^{14-17}$ Fullterm neonates do not produce melatonin for 3-5 months leading to transient melatonin deficiency and this deficiency is more prolonged in premature neonates. ${ }^{18}$ Evidence from several studies suggests a protective role for melatonin in perinatal diseases including RDS, asphyxia and sepsis. ${ }^{6,10,19,20}$ In this study, we aimed to evaluate the effectiveness of melatonin for the prevention of BPD in preterm infants with RDS.

\section{Material and Methods}

This randomized clinical trial was conducted in AL Zahra hospital which is a tertiary, university referral center in the North West of Iran from March 2019-April 2020. The study was approved by the Ethics committee of Tabriz University of Medical Sciences by code IR.TBZMED.REC. 1398.574 at 2019.08.19 and registered in the Iranian Registry of Clinical Trials (IRCT) by the number IRCT 20190518043629N21. Parental informed written consent was obtained before patient enrollment. Based on the study by Gitto et al. ${ }^{10}$ considering reducing the rate of mortality or BPD from $66 \%$ to $42 \%$ in the intervention group with power of $80 \%$ and alpha 0.05 we estimate that 40 cases were needed for each group. Inborn preterm infants with RDS who needed surfactant replacement therapy and had a gestational age of 27-32 weeks and a birth weight less than 1250 grams were eligible for the study. Exclusion criteria were major congenital anomalies, birth asphyxia (Apgar score $\leq 3$ at the first minute of birth), major cardiac diseases (not including patent ductus arteriosus), chromosomal anomalies, abdominal distention or evidence for necrotising enterocolitis at first hours of birth, and parental refuse. Infants who need endotracheal intubation at birth due to ineffective respiratory drive were excluded from the study. Our center policy is stabilization of the infant by using continuous positive airway pressure (CPAP) for preterm neonates in the delivery room and saving intubation only for those who have apnea or ineffective respiration.

CPAP was administered through short bilateral nasal prongs, intermittently with a nasal mask. Distending pressure was generated by a variable flow nasal CPAP device and positive end-expiratory pressure (PEEP) 5-6 cm H2O and flow 6-7 litter/min (Fisher\& Paykel Health Care limited, New Zealand). Patients with evidence of respiratory distress (tachypnea, retractions, and/or nasal flaring) shortly after delivery, a persistent oxygen requirement with $\mathrm{FiO} 2$ more than 0.3 and radiographic findings of RDS received surfactant within 6 hours of birth. Enrolled patients were randomly allocated in two groups by random number list generated by random number generator in sequentially numbered, opaque, sealed and stapled envelopes. Curosurf® (Poractant alpha, Chiesi Farmaceutici, Italy) $200 \mathrm{mg} / \mathrm{kg} / \mathrm{dose}(2.5$ $\mathrm{ml} / \mathrm{kg}$ /dose intra-tracheally) with the INSURE technique was used in group A. Patients in group $B$ received melatonin (Nature Made, Pharmavit, USA) $5 \mathrm{mg} / \mathrm{kg} /$ day per gastric tube for 3 days in addition to Curosurf®. After surfactant was administered, when the spontaneous respirations resumed, and adequate heart rate 
and oxygen saturation was established, the endotracheal tube was removed and the infants were weaned to nasal-CPAP. Arterial blood gas parameters were recorded at admission and at 6 hour intervals after surfactant administration. The primary outcome was the development of BPD. The secondary outcome was considered other complications of prematurity, such as the duration of hospital stay and mortality. BPD was defined as the need for supplemental oxygen for at least 28 days and its severity determined at 36 weeks of gestation age based on the fraction of inspired oxygen. ${ }^{11}$ Cranial ultrasound examination was performed on days 5 to 7 of birth for the diagnosis of intraventricular hemorrhage (IVH) by an experienced pediatric radiologist. Patent ductus arteriosus (PDA) was diagnosed based on clinical signs and confirmed by echocardiography performed by an expert pediatric cardiologist. Pneumothorax was determined by the presence of air in pleural space on chest X-ray. Necrotizing enterocolitis was suspected in neonates with abdominal distention and feeding intolerance. This diagnosis was confirmed by the presence of gas or air bubbles in the wall of the intestine on an abdominal X-ray and its severity was determined by Bell's staging system. ${ }^{21}$

Eye examination for the diagnosis of retinopathy of prematurity (ROP) was done through indirect ophthalmoscopy by an expert opthalmologist who was blind about patients groups.

Another independent researcher who was blind about patients groups completed a detailed questionnaire.

Statistical analyses were performed using the statistical package for social sciences (SPSS) version 17.0. Quantitative data were presented as mean \pm standard deviation (SD) and qualitative data as frequency and percent. Independent $t$ test were used for testing continuous normally distributed data. Categorical data were compared between groups using Chi- square or Fisher exact test. Two tailed tests were used and a p. value less than 0.05 was considered statistically significant.

\section{Results}

A total of 120 preterm infants with RDS were admitted to the neonatal intensive care unit (NICU) between March 2019 and April 2020. Ninty four infants met inclusion criteria with respect to their gestation age and birth weight. Five neonates were excluded from the study because of major congenital anomalies, 5 cases were intubated before arrival to the NICU and 4 patients because parents refused to consent. A total of 80 neonates were enrolled in the study including forty neonates in the surfactant group (group A) and 40 in the surfactant and melatonin group (group B). The mean gestation age and birth weight of studied patients were $31.3 \pm 3.8$ wks and $1189 \pm 84$ grams, respectively. Thirty-five $(43.8 \%)$ patients were girls and 45 (56.2\%) were boys. Demographic characteristics of patients are shown in Table I.

The number of patients to develop BPD was statistically significantly less in the group that received both surfactant and melatonin $(n=24$, $(60 \%)$ in group $\mathrm{A}, \mathrm{n}=18(\% 45) \mathrm{p}=0.02)$.

The duration of hospital stay, need for mechanical ventilation and mortality were significantly lower in patients in group B. The complications and outcomes of patients in both groups are shown in Table II.

Maximal $\mathrm{FiO}_{2}$ at the first hour and $4 \mathrm{hrs}$ after surfactant replacement therapy was 0.37 and 0.35 in group A and 0.35 and 0.34 in group B respectively, without significant difference.

No adverse drug reaction was observed in patients receiving melatonin.

\section{Discussion}

In this study, administration of melatonin in addition to surfactant in comparison to surfactant replacement therapy alone decreased the duration of mechanical ventilation and hospital stay significantly. The incidence of BPD, PDA, IVH, air leak syndrome and mortality were reduced in neonates who received melatonin. 
Table I. Demographic characteristics of study groups.

\begin{tabular}{|c|c|c|c|}
\hline & $\begin{array}{c}\text { Group A } \\
\mathrm{N}=40\end{array}$ & $\begin{array}{c}\text { Group B } \\
\mathrm{N}=40\end{array}$ & $\mathrm{P}$ value \\
\hline Gestation age, weeks & $29.3 \pm 2.9$ & $27.9 \pm 3.9$ & NS \\
\hline Birth weight, gr & $1154 \pm 98$ & $1112 \pm 124$ & NS \\
\hline Maternal age, years & $30.1 \pm 3.1$ & $31.8 \pm 4.2$ & NS \\
\hline Gender & & & NS \\
\hline Male, n(\%) & $22(55)$ & $23(47.5)$ & \\
\hline Previous preterm labor $\mathrm{n}(\%)$ & $3(7.5)$ & $4(10)$ & NS \\
\hline \multicolumn{4}{|l|}{ Apgar score } \\
\hline 1st minute & $6.1 \pm 1.7$ & $5.9 \pm 1.7$ & NS \\
\hline 5th minute & $8.3 \pm 1.02$ & $8.4 \pm 1.02$ & NS \\
\hline
\end{tabular}

$\mathrm{NS}=$ not significant

Table II. Comparision of complications in both groups.

\begin{tabular}{|c|c|c|c|c|}
\hline & $\begin{array}{c}\text { Group A } \\
\mathrm{N}=40\end{array}$ & $\begin{array}{c}\text { Group B } \\
\mathrm{N}=40\end{array}$ & $\mathrm{P}$ value & $\mathrm{RR}(95 \% \mathrm{CI})$ \\
\hline Duration of $\mathrm{MV}, \mathrm{d}$ & $7.6 \pm 2.1$ & $5.3 \pm 1.6$ & 0.003 & $(0.1-3.8) 2$ \\
\hline Duration of hospital stay, days & $10.7 \pm 2.7$ & $6.4 \pm 1.5$ & 0.02 & $(1.5-2.1) 1.5$ \\
\hline $\mathrm{BPD}, \mathrm{n}(\%)$ & $24(60)$ & $18(45 \%)$ & 0.02 & $(0.1-6.23) 2$ \\
\hline $\mathrm{IVH}, \mathrm{n}(\%)$ & $16(40)$ & $12(30)$ & 0.04 & $(0.1-9.3) 1.5$ \\
\hline PDA, n(\%) & $9(22.5)$ & $5(15)$ & 0.03 & $(0.1-76.7) 1.5$ \\
\hline Air leak syndroms, n(\%) & $4(10)$ & $2(5)$ & 0.01 & \\
\hline Pulmonary hemorrhage, n (\%) & $3(7.5)$ & $3(7.5)$ & 1 & \\
\hline Mortality , n (\%) & $6(15)$ & $3(7.5)$ & 0.009 & $(0.1-8.6) 2$ \\
\hline
\end{tabular}

$\mathrm{MV}=$ mechanical ventilation, $\mathrm{BPD}=$ bronchopulmonary dysplasia, $\mathrm{IVH}=$ intraventricular hemmorhagea, $\mathrm{PDA}=$ patent ductus arteriosus

Lung injury in the neonatal period is mediated by several etiologic factors such as genetic, hemodynamic, metabolic, nutritional, mechanical, and infectious or inflammatory mechanisms. All these events lead to an increased synthesis of free radicals which, in turn, induces oxidative stress-mediated tissue damage that is involved in the development of BPD. ${ }^{18}$

Numerous reports have demonstrated that melatonin is a broad-spectrum antioxidant. ${ }^{22}$ Pan and et al. ${ }^{23}$ demonstrated that the nocturnal administration of melatonin reduced interstitial fibrosis and the total number of alveoli caused by BPD. There are reports showing inflammatory cytokines are elevated in preterm newborns who develop BPD. ${ }^{24,25}$ Gitto and et al. ${ }^{26}$ showed melatonin treatment reduced the proinflammatory cytokines and improved clinical outcome. Melatonin stimulates several antioxidant enzymes in addition to its direct scavenging actions without pro-oxidant effects.

We did not observe any side effects in our patients due to melatonin. Melatonin appears safe and no side effects have been reported when $100 \mathrm{mg} / \mathrm{kg}$ was administered in 54 hours or $10 \mathrm{mg} / \mathrm{kg}$ once daily for 5 days. ${ }^{27,28}$ Carloni et al. ${ }^{29}$ used three different oral doses of melatonin: $0.1,0.5$ and $5 \mathrm{mg} / \mathrm{kg}$ in neonates born before 37 weeks of gestation. They found that a single intragastric administration of these doses resulted in a high peak plasma concentration that indicates that it is well absorbed after intragastric bolus. These high concentrations 
appear not to be dose-dependent and are higher than what was found after oral administration in adults.

The limitations of this study was the small sample size, lack of long-term follow-up of studied patients and the lack of paraclinical data about proinflammatory cytokines.

In conclusion, the present study showed that melatonin administration as adjuvant therapy in preterm infants with RDS is associated with better outcomes with respect to duration of hospital stay, mortality, need for mechanical ventilation and complications of prematurity such as BPD, air leak syndromes, IVH and PDA. Future studies are recommended to establish the appropriate melatonin dose and timing of administration to achieve the best clinical outcome.

\section{Acknowledgement}

This study was supported by the Pediatric Health Research Center. We thank the NICU nurses involved in the care of study infants. We also thank Mrs. Namdari for her valuable contribution.

\section{Ethical approval}

The study was approved by the Ethic committee of Tabriz University of Medical Sciences by code IR.TBZMED.REC. 1398.574 at 2019.08.19 and registered in Iranian Registry of Clinical Trials (IRCT) by number IRCT 20190518043629N21.

\section{Author contribution}

The authors confirm contribution to the paper as follows: study conception and design: MMG, ES; data collection: MF; analysis and interpretation of results: MMG, SY; draft manuscript preparation: MMG, MF. All authors reviewed the results and approved the final version of the manuscript.

\section{Source of funding}

Funding support by Pediatric Health Research Center. Tabriz University of Medical Sciences

\section{Conflict of interest}

The authors declare that there is no conflict of interest.

\section{REFERENCES}

1. Rieter RJ, Pardes SD, Manchester LC, Tan DX. Reducing oxidative/nitrosative stress: a newly discovered gene for melatonin. Crit Rev Biochem Mol Biol 2009; 44: 175-200. https://doi. org/10.1080/10409230903044914

2. Radogna F, Diederich M, Chibelli L. Melatonin: a pleiotropic molecule regulating inflammation. Biochem Pharmacol 2010; 80: 1844-1852. https://doi. org/10.1016/j.bcp.2010.07.041

3. Hardeland R, Cardinali DP, Srinivasan V, Spence DW, Brown GM, Pandi-Perumal SR. Melatonin- a pleitropic, orchestrating regulator molecule. Prog Neurobiol 2011; 93:350-384. https://doi.org/10.1016/j. pneurobio.2010.12.004

4. Reiter RJ, Tan DX, Osuna C, Gitto E. Actions of melatonin in the reduction of oxidative stress: a review. J Biomed Sci 2000; 7: 444-448. https://doi. org/10.1007/BF02253360

5. Reiter RJ, Tan DX, Burkhardt S. Reactive oxygen and nitrogen species and cellular and organismal decline: ameloration with melatonin. Mech Aging Dev 2002; 123: 1007-1019. https://doi.org/10.1016/ S0047-6374(01)00384-0

6. Gitto E, Aversa S, Reiter RJ, Barberi I, Pellegrino S. Update on the use of melatonin in pediatrics. J Pineal Res 2011; 50: 21-28. https://doi.org/10.1111/ j.1600-079X.2010.00814.x

7. Owens JA, Witamans M. Sleep problems. Curr Probl Pediatr Adolesc Health Care 2004; 34: 154-179. https://doi.org/10.1016/j.cppeds.2003.10.003

8. Paproka J, Dec R, Jamroz E, Marszal E. Melatonin and childhood refractory epillepsy- a pilot study. Med Sci Monit 2010; 16: CR389-CR396.

9. Saugstad OD. Oxidative stress in the newborn- a 30 year perspective. Biol Neonate 2005; 88: 228-236. https://doi.org/10.1159/000087586 
10. Gitto E, Reiter RJ, Amodio A, et al. Early indicators of chronic lung disease in preterm infants with respiratory distress syndrome and their inhibition by melatonin. J Pineal Res 2004; 36: 250-255. https:// doi.org/10.1111/j.1600-079X.2004.00124.x

11. Jobe AH, Bancalari E. Bronchopulmonary dysplasia. Amer J Respir Crit Care Med 2001; 163: 1723-1729. https://doi.org/10.1164/ajrccm.163.7.2011060

12. Bhandari A, Bhandari V. Pitfalls, problems, and progress in bronchopulmonary dysplasia. Pediatrics 2009; 123: 1562-1573. https://doi.org/10.1542/ peds.2008-1962

13. Halliwell B. Free radicals, antioxidants and human disease: curosity, cause or consequences? Lancet 2009; 344: 721-724. https://doi.org/10.1016/S01406736(94)92211-X

14. Laughon MM, Smith PB, Bose C. Prevention of bronchopulmonary dysplasia. Seminars Fetal Neonat Med 2009; 14: 374-382. https://doi. org/10.1016/j.siny.2009.08.002

15. Almario B, Wu S, Peng J, Alapati D, Chen S, Sosenko IR. Pentoxifylline and prevention of hyperoxiainduced lung -injury in neonatal rats. Pediatr Res 2012; 71: 583-589. https://doi.org/10.1038/pr.2012.14

16. Tropea K, Christou H. Current pharmacologic approaches for prevention and treatment of bronchopulmonary dysplasia. Inter J Ped 2012; 2012: 598606. https://doi.org/10.1155/2012/598606

17. Gharehbaghi MM, Peirovifar A, Ghojazadeh M, Mahallei M. Efficacy of azithromycin for prevention of bronchopulmonary dysplasia.Turk J Med Sci 2012; 42: 1070-1075.

18. Gitto E, Marseglia L, Manti S, et al. Protective role of melatonin in neonatal diseases. Oxid Med Cell Longev 2013; 2013: 980374. https://doi. org/10.1155/2013/980374

19. Ahmad QM, Chishti AL, Waseem N. Role of melatonin in management of hypoxic ischaemic encephalopathy in newborns: A randomized control trial. J Pak Med Assoc 2018; 68: 1233-1237.

20. Henderson R, Kim S, Lee E. Use of melatonin as adjunctive therapy in neonatal sepsis: a systematic review and meta analysis. Complemen Ther Med 2018; 39: 131-136. https://doi.org/10.1016/j. ctim.2018.06.002
21. Walsh MC, Kliegman RM. Necrotizing enterocolitis: treatment based on staging criteria. Pediatr Clin North Am 1986; 33: 179-201. https://doi.org/10.1016/ S0031-3955(16)34975-6

22. D'Angelo G, Chimenz R, Reiter RJ, Gitto E. Use of Melatonin in Oxidative Stress Related Neonatal Diseases. Antioxidants (Basel) 2020; 9: 477. https:// doi.org/10.3390/antiox9060477

23. Pan L, Fu JH, Xue XD, Xu W, Zhou P, Wei B. Melatonin protects against oxidative damage in a neonatal rat model of bronchopulmonary dysplasia. World J Pediatr 2009; 5: 216-221. https://doi. org/10.1007/s12519-009-0041-2

24. Vento G, Mele MC, Mordente A, et al. High total antioxidant activity and uric acid in tracheobronchial aspirate fluid of preterm infants during oxidative stress: an adaptive response to hyperoxia?. Acta Paediatr 2000; 89: 336-342. https:// doi.org/10.1111/j.1651-2227.2000.tb01336.x

25. Balany J, Bhandari V. Understanding the impact of infection, inflammation, and their persistence in the pathogenesis of bronchopulmonary dysplasia. Front Med (Lausanne) 2015; 2: 90. https://doi.org/10.3389/ fmed.2015.00090

26. Gitto E, Reiter RJ, Sabatino G, et al. Correlation among cytokines, bronchopulmonary dysplasia and modality of ventilation in preterm newborns: improvement with melatonin treatment. J Pineal Res 2005; 39: 287-293. https://doi.org/10.1111/j.1600079X.2005.00251.x

27. Gitto E, Romeo C, Reiter RJ, et al. Melatonin reduces oxidative stress in surgical neonates. J Pediatr Surg 2004; 39: 184-189. https://doi.org/10.1016/j. jpedsurg.2003.10.003

28. Aly H, Elmahdy H, El-Dib M, Rowisha M, El- Gohary T, Elbatch M. Melatonin use for neuroprotection in perinatal asphyxia: a randomizes controlled pilot study. J Perinatol 2015; 35: 186-191. https://doi. org/10.1038/jp.2014.186

29. Carloni S, Proietti F, Rocchi M, et al. Melatonin pharmacokinetics following oral adminstration in preterm neonates. Molecules 2017; 22: 2115. https:// doi.org/10.3390/molecules22122115 